\title{
A Randomized Trial of Wearable UV dosimeter for Skin Cancer Prevention
}

\author{
George I Varghese, M.D. ${ }^{1}$, Peter D Kaplan, Ph.D. ${ }^{2}$, Catherine Do, M.D., Ph.D., ${ }^{3}$ Melissa Barrer, \\ M.M.S., ${ }^{1}$ Khaled Ezzedine, M.D., Ph.D., ${ }^{4}$ Jonathan H Zippin, M.D., Ph.D., ${ }^{1}$ Emmanuel LP Dumont, \\ Ph.D. ${ }^{2,5, *}$ \\ ${ }^{1}$ Department of Dermatology, Weill Cornell Medicine, New York, New York \\ 2 Shade, 111 Ideation Way, Nutley, NJ \\ ${ }^{3}$ Department of Pathology, New York University Langone Health, 22nd Floor, 240 East 38th Street, \\ New York, NY 10016 \\ ${ }^{4}$ University Hospital Henri Mondor, Department of Dermatology, 51 Avenue du Maréchal de Lattre de \\ Tassigny, 94010 Créteil, France \\ ${ }^{5}$ Hackensack Meridian Center for Discovery and Innovation, 111 Ideation Way, Nutley, NJ \\ * correspondence to: em@shade.io
}

\section{Abstract}

Background

Non-melanoma skin cancer (NMSC) is the most prevalent cancer in the United States, affecting 5 million people and costing $\$ 8.1$ billion per year. Despite well-defined guidelines on ultraviolet radiation (UVR) avoidance, it remains difficult for people to assess their individual exposure, as UVR is invisible and the onset of symptoms due to UVR damage are delayed.

\section{Methods}

In a prospective, randomized-controlled trial, 97 patients with a history of actinic keratoses were enrolled from March 2018 to July 2018 and followed over 6 months. 50 patients were given a wearable device that measured UV exposure and a smartphone application that provided both real-time and cumulative UV exposure information, and 47 patients were provided with UV protection counseling by a dermatologist.

Results

We observed a significant decrease in the incidence rate of NMSC in the intervention group compared to the control group over 3 months $(p=0.02)$. We did not observe a significant decrease in the incidence rate of actinic keratoses $(\mathrm{AK})$ in the intervention group compared to the control group. The observed clinical benefit was not accompanied by psychological side effects such as anxiety and depression.

\section{Conclusions}

This study suggests that providing real-time UV exposure data using a wearable UV dosimeter is a safe and effective behavioral change strategy to prevent NMSC. (Funded by the National Cancer Institute, contract HHSN261201700005C; ClinicalTrials.gov number NCT03315286.) 
Skin cancer is the most common cancer in the United States and its incidence is still on the rise worldwide. ${ }^{1}$ Genetic, phenotypic and environmental factors, specifically ultraviolet radiation (UVR), are considered the largest contributing factors to the development of skin cancer. ${ }^{2}$ Over the past three decades, there has been a push towards protecting the skin from the dangers of UVR through educational campaigns about the harmful effects of UVR, ${ }^{3}$ topical application of physical and chemical blockers in sunscreens, ${ }^{4}$ ultraviolet (UV) protective clothing, ${ }^{5}$ and vitamin supplementation such as niacinamide. ${ }^{6}$ More recent controversies on the effectiveness ${ }^{7}$ and safety ${ }^{8}$ of sunscreens have created a critical need for safer strategies to help reduce the overall UVR exposure to our skin. UVR dosimeters provide a data-driven solution for assessing and communicating the real-time risk of UVR to support immediate protective behavior. The Shade ${ }^{\circledR}$ UV sensor has the ability to accurately record UVI and has reached standard benchmarks making it superior to other wearable dosimeters. ${ }^{9,10}$ In this prospective, randomized clinical trial, we assessed the clinical efficacy of the Shade UV sensor in a patient population with a history of actinic keratoses.

METHODS

\section{Trial oversight}

This prospective, randomized, observer-blinded, controlled clinical trial enrolled patients with a history of actinic keratoses at a single site in New York City, NY. The trial was conducted in accordance with the Institutional Review Board (IRB) of Weill Cornell Medicine, the National Cancer Institute (NCI) of the National Institutes of Health, and applicable governmental regulations. The IRB and the $\mathrm{NCI}$ approved the protocol and the consent forms. All participants provided written informed consent before enrollment. The sponsor, YouV Labs, Inc., and the PI of the trial (GV) were responsible for the overall trial design (with input from the IRB and the $\mathrm{NCl}$ ), site selection, monitoring, and data analysis. Investigators were responsible for data collection, recruitment, and treatment. The authors vouch for the accuracy and completeness of the data and for the fidelity of the trial to the protocol. The trial was registered on clinicaltrials.gov under the identification NCT03315286.

\section{Participants, randomization, and data blinding}

Eligible participants were persons 18 years of age or older with a history of actinic keratosis (AKs, one diagnosis in the 12 months prior to enrollment or $5 \mathrm{AKs}$ in the 5 years prior to enrollment). Patients having received UV therapy in the past 6 months or field therapy for the treatment of actinic keratosis in the past 3 months were excluded. Participants were assigned using randomly-generated blocks of four, stratified by skin type, to receive a wearable UV dosimeter or standard of care UVR education. The randomization in blocks of 4 patients was used to balance seasonal trends in UV exposure. Participants receiving a dosimeter were encouraged to wear it every day and received compliance payments if they used it at least two days per week. At enrollment, all participants from both groups were advised to minimize their UV exposure using sunscreen and protective clothing. Participants were enrolled from April to July 2018 and had two follow-up visits at 3 month intervals. The final visits ran from November to January 2019. All participants from both groups were examined by the same dermatologist who was blinded to their group assignment.

\section{Wearable UV dosimeters}

The sponsor provided the Shade UV dosimeters and a companion smartphone application. The dosimeters were designed to measure the UVI, a real-time measure of the strength of UVR relevant to skin health. The dosimeter's range of detection is from $0.01 \mathrm{UVI}$ to $12 \mathrm{UVI}$, ensuring that sensors pick 
up UV in low-UV situations and do not saturate during the summer. Its accuracy was evaluated across New York and Florida in a variety of sunlight situations. ${ }^{10}$ The dosimeters measured the UVI every second and aggregated the cumulative dose every 6 minutes. They could function for about 5 days on a single charge. They were rechargeable with a provided micro-USB cable and a wall charger. Dosimeters vibrated when covered and had an LED to indicate charging progress. Finally, they were designed to be worn on the chest using a magnetic attachment (See Figure 1).

The sponsor developed an application for both Apple and Android smartphones that connected to the UV dosimeter via Bluetooth. The applications were developed specifically for the trial and were not updated during the trial. Once paired to a Shade UV dosimeter, the smartphone application displayed a real-time UV index, real-time cumulative UV exposure, as well as historical data of daily UV exposure. At enrollment, participants receiving the dosimeter were trained to use the dosimeter and select a daily UV dose threshold on the application. This threshold was customizable at any time through the application and was used to send notifications to alert participants on their UV dose. Participants could also input their sunscreen use into the application and the cumulative UV exposure would stop increasing during the two hours following sunscreen application.
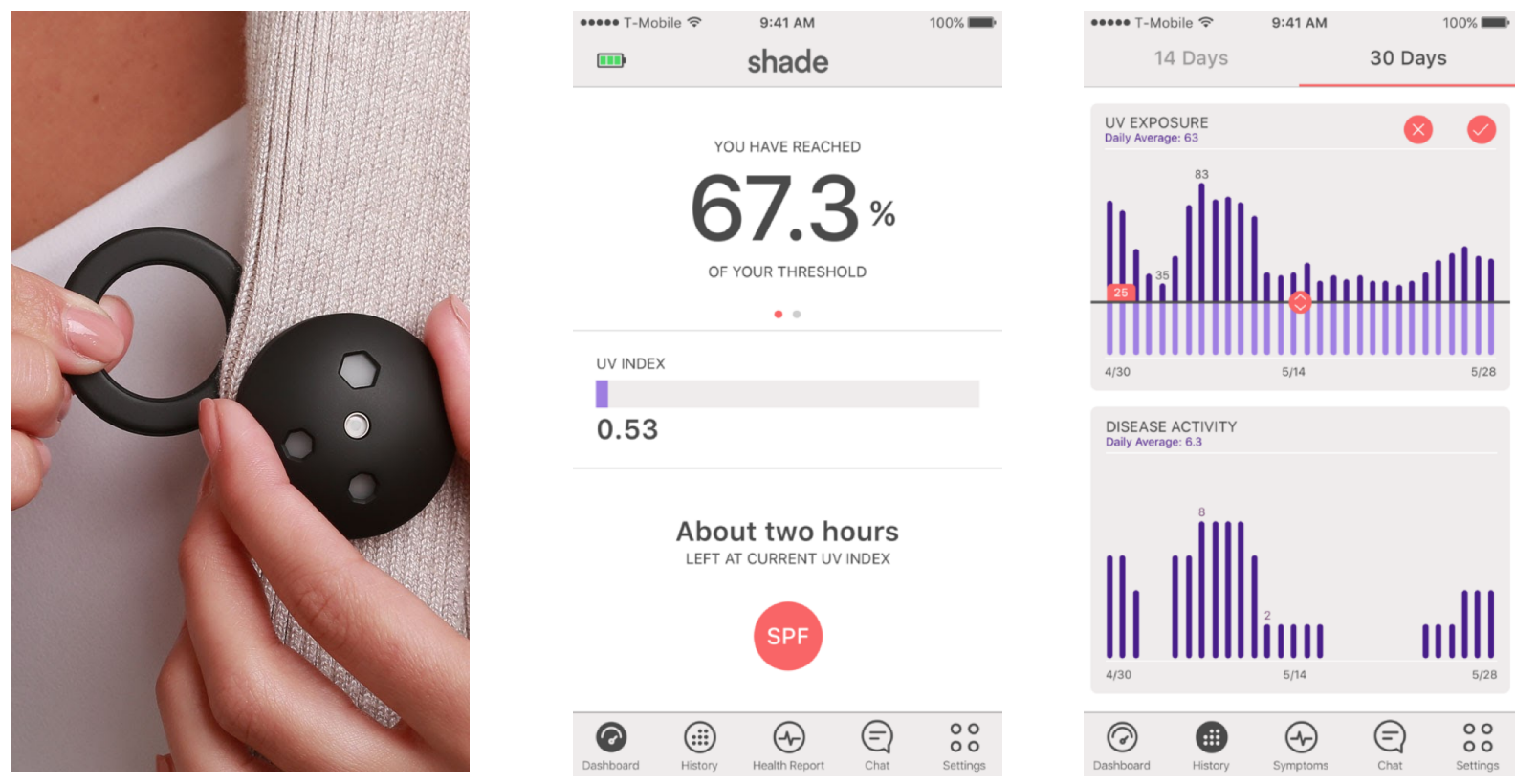

Figure 1. Wearable UV dosimeter, its magnet, and its companion smartphone application.

\section{Safety assessments}

Safety assessments included monitoring of adverse events related or possibly related to the device or sun exposure experienced within the study period. Adverse events were to be reported to the clinical principal investigator, the study coordinator, the IRB, and the $\mathrm{NCl}$.

\section{Efficacy assessments}

AK lesions and NMSC on sun-exposed skin areas (scalp, face, hands) were counted and reported by a single, blinded dermatologist at enrollment, and at each subsequent visit (three months after enrollment and six months after enrollment). Pictures of every lesion and their locations were recorded. AKs are defined as a keratotic macule(s) or papule(s) on an erythematous base. To ensure that only new AKs or NSMCs after enrollment were counted, each lesion's location and picture were compared to prior lesions (AK or NSMC). The primary endpoint was the efficacy of the UV dosimeter 
in preventing the formation of AKs at disenrollment compared to the intermediary visit. AKs are common skin lesions induced by sun exposure. ${ }^{11,12}$ Secondary clinical endpoints included the efficacy of the Shade UVR sensor in preventing the formation of non melanoma skin cancers (squamous cell carcinoma (SCC) and basal cell carcinoma (BCC) combined), SCC alone, BCC alone, and melanoma. All lesions suspected of being cancerous were biopsied and the diagnosis was confirmed by a blinded pathologist at Weill Cornell Medicine. Other secondary endpoints included scores on surveys of anxiety, depression, and the ability to participate in social activities, as measured by the National Institute of Health's Patient-Reported Outcomes Measurement Information System (PROMIS).

\section{Data entry}

Case Report Forms (CRFs) were filled out by participants, the study coordinator, and the dermatologist on paper. CRF's were monitored for completeness, consistency, and agreement with underlying medical records periodically during the study. CRFs were scanned and data entered by two people. Discrepancies between the two entries were reconciled manually.

\section{Statistical analysis}

Incidence rates (IR) of AK and NMSC within 3-month intervals at the intermediary visit and disenrollment were calculated using poisson models. The IR at the intermediary visit was considered as baseline since the lesions observed at enrollment resulted from an undefined amount of exposure time before enrollment. The incidence rate ratio (IRR) between groups was calculated using a longitudinal approach comparing the differences in IRs from the intermediary visit to disenrollment in each group (The IR is the slope estimated by the interaction term between time and group assignment in the poisson model). To control for confounding factors, we used a multivariate poisson model including any clinico-demographic variables with a significant or sub-significant difference between groups at baseline $(p$-value<0.1). Categorical variables were tested using Chi-squared test or Fisher's exact test (when the chi-squared test requirements were not met) and continuous variables using Welch t-tests or non parametric Spearman tests (when the variable was not normally distributed). Normality was tested using skewness and kurtosis tests for normality. All p-values were computed using two-tailed tests and performed using STATA ${ }^{\circledR}$ v13 (STATA corp).

\section{RESULTS}

\section{Trial population}

Between April 1, 2018, and July 31, 2018, a total of 97 patients underwent randomization. 50 were assigned to the device group and received a Shade UV sensor and UV protection counseling, and 47 were assigned to the control group and received UV protection counseling only (Figure 2). Following enrollment, 1 patient in the device group was excluded from the per-protocol population for missing a protocol visit and 4 patients in the control group were excluded from the per-protocol population for either lack of compliance with treatment protocol (1) or for missing protocol visits (3). Skin type, defined by the Fitzpatrick scale (from 1 to 6 ), ${ }^{13}$ was balanced between the device and the control group (Table 1). Participants with a Fitzpatrick skin type 1 or 2 accounted for $92 \%$ of the participants and the remaining $8 \%$ had a Fitzpatrick skin phototype 3 . Gender, skin type, ethnicity, race, education, and known skin cancer risk factors were balanced in the two groups (cf. Table 1). All participants reported their race to be White. The mean age of the participants was 66 years. Despite randomization, the participants in the device group were significantly younger than the participants in 
the control group, by 5 years on average. The imbalance in age was accounted for in the statistical analysis.

\section{Safety}

No adverse events were reported during the trial.

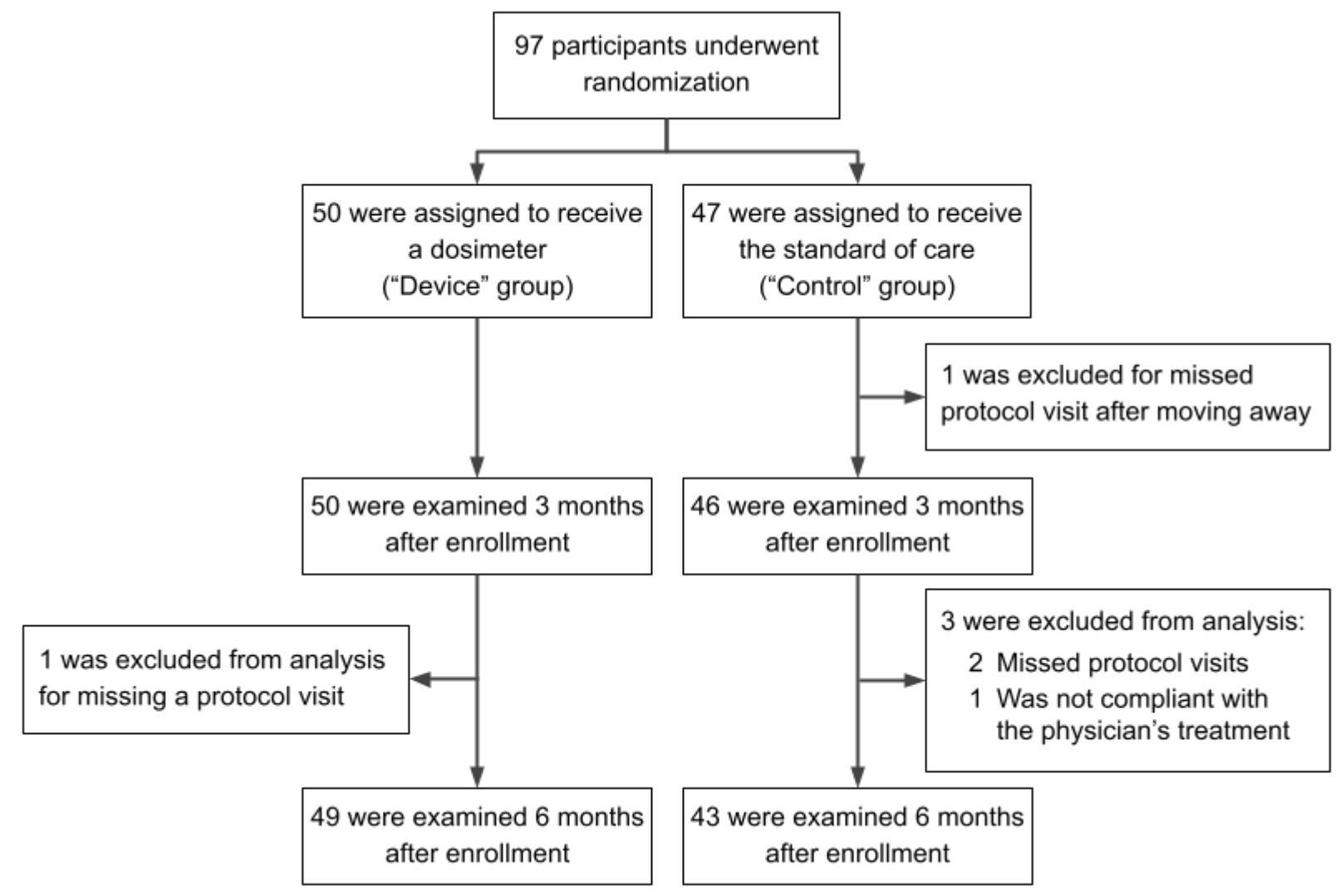

Figure 2. Randomization and Analysis Populations.

\section{Efficacy}

In Table 2, we present the number of lesions (AK and NMSC) at enrollment (before summer), at the intermediary visit (3 months after enrollment), and disenrollment (after summer, 6 months after enrollment). The incidence rate ratio was calculated using a Poisson regression analysis after controlling for gender and for age. We measured an IRR of 0.799 ( $p$-value $=0.44,95 \% \mathrm{Cl}$ : 0.450 1.415 ) for AKs and an IRR of 0.05 ( $p$-value $=0.024,95 \% \mathrm{Cl}: 0.004-0.67)$ for NMSC. Among the NIH's PROMIS questionnaires, with scores ranging from 8 to 40, we selectively assessed 3 relevant psycho-social areas, depression, anxiety and the ability to participate in social events. We found a relative decrease of 2.1 points ( $p$-value $=0.010,95 \% \mathrm{Cl}:-3.69,-0.50$ ) in the ability to participate in social events. 
Table 1. Demographic and clinical characteristics.

\begin{tabular}{|c|c|c|c|c|}
\hline Characteristics & $\begin{array}{l}\text { Control } \\
(\mathrm{N}=43)\end{array}$ & $\begin{array}{l}\text { Device } \\
(\mathrm{N}=49)\end{array}$ & $\begin{array}{c}\text { Total } \\
(\mathbf{N}=92)\end{array}$ & P-value \\
\hline Gender - no. of participants (\%) & & & & 0.119 \\
\hline Male & $24(56 \%)$ & $35(71 \%)$ & $59(64 \%)$ & \\
\hline Female & $19(44 \%)$ & $14(29 \%)$ & $33(36 \%)$ & \\
\hline Mean age (SD) - yr & $69(7.0)$ & $64(10)$ & $66(9)$ & $0.0001\left(^{*}\right)$ \\
\hline $\begin{array}{l}\text { Diagnosed with NMSC at enrollment - no. of } \\
\text { participants (\%) }\end{array}$ & & & & 0.49 \\
\hline No & $39(91 \%)$ & $41(84 \%)$ & $80(87 \%)$ & \\
\hline Yes & $4(9 \%)$ & $8(16 \%)$ & $12(13 \%)$ & \\
\hline Race - no. of participants (\%) & & & & $\mathrm{n} / \mathrm{a}$ \\
\hline White & $43(100 \%)$ & $49(100 \%)$ & $92(100 \%)$ & \\
\hline Not White & $0(0 \%)$ & $0(0 \%)$ & $0(0 \%)$ & \\
\hline Ethnicity - no. of participants (\%) & & & & 0.494 \\
\hline Hispanic or Latino & $0(0 \%)$ & $2(4 \%)$ & $2(2 \%)$ & \\
\hline Not Hispanic or Latino & $38(88 \%)$ & $39(80 \%)$ & $77(84 \%)$ & \\
\hline Unknown & $5(12 \%)$ & $8(16 \%)$ & $13(14 \%)$ & \\
\hline Fitzpatrick type - no. of participants (\%) & & & & 0.429 \\
\hline Type 1 & $11(26 \%)$ & $19(39 \%)$ & $30(33 \%)$ & \\
\hline Type 2 & $27(63 \%)$ & $26(53 \%)$ & $53(58 \%)$ & \\
\hline Type 3 & $5(12 \%)$ & $4(8 \%)$ & $9(8 \%)$ & \\
\hline Education - no. of participants (\%) & & & & 0.931 \\
\hline Did not complete college & $5(12 \%)$ & $6(12 \%)$ & $11(12 \%)$ & \\
\hline Completed college & $37(86 \%)$ & $42(86 \%)$ & $79(86 \%)$ & \\
\hline Unknown & $1(2 \%)$ & $1(2 \%)$ & $2(2 \%)$ & \\
\hline \multicolumn{5}{|l|}{ Risk factor for skin cancer - no. of participants (\%) } \\
\hline $\begin{array}{l}\text { Had phototherapy in the } 6 \text { months prior to } \\
\text { enrollment }\end{array}$ & $0(0 \%)$ & $1(2 \%)$ & $1(1 \%)$ & 1.000 \\
\hline Current smoker & $1(2 \%)$ & $1(2 \%)$ & $2(2 \%)$ & 1.000 \\
\hline Regular user of a tanning bed & $1(2 \%)$ & $0(0 \%)$ & $1(1 \%)$ & 0.467 \\
\hline
\end{tabular}

$\mathrm{SD}=$ Standard Deviation. All demographic characteristics were reported by the participant except for the Fitzpatrick skin type, reported by the clinical principal investigator. For categorical covariates, p-values were calculated using Chi-square tests (gender and education) and Fisher's exact tests, when the Chi-squared test requirement was not met (ethnicity, smoking status, skin type, use of a tanning bed, and history of phototherapy). We used a Spearman t-test for the age. 


\begin{tabular}{lcc}
\hline & $\begin{array}{c}\text { Control } \\
(\mathbf{n = 4 3 )}\end{array}$ & $\begin{array}{c}\text { Device } \\
(\mathbf{n}=\mathbf{4 9})\end{array}$ \\
\hline $\begin{array}{l}\text { Actinic Keratoses } \\
\text { lesions at enrollment (mean) }\end{array}$ & $304(6.2)$ \\
lesions at 3 months (mean) & $271(6.5)$ & $263(5.2)$ \\
lesions at 6 months (mean) & $198(4.5)$ & $194(4.0)$ \\
IRR [95\% Cl] b/w 6 months and 3 months & $183(4.2)$ & $0.799[0.450,1.415]$ \\
p-value & $n / a$ & 0.44 \\
\hline Non-melanoma Skin Cancers & $n / a$ & $12(0.24)$ \\
lesions at enrollment (mean) & & $4(0.08)$ \\
lesions at 3 months (mean) & $5(0.11)$ & $2(0.04)$ \\
lesions at 6 months (mean) & $1(0.02)$ & $0.05[0.004,0.67]$ \\
IRR [95\% Cl] b/w 6 months and 3 months & $10(0.23)$ & $0.024\left(^{*}\right)$ \\
p-value & $n / a$ & $n / a$ \\
\hline
\end{tabular}

Table 2. Skin lesions diagnosed at disenrollment after summer. The incidence rate ratio (IRR) was computed using a Poisson model adjusted for gender and age between the final visit at 6 months and the intermediary visit at 3 months. $\mathrm{Cl}=$ Confidence Interval.

\section{DISCUSSION}

This randomized clinical trial was designed to evaluate a novel sun protection strategy where real-time, accurate UV information is provided to participants without specific recommendations on UV dose limits. Our study was powered to detect a $25 \%$ reduction in the rate of newly-formed AKs over one summer. AKs are an ideal outcome measure in a study evaluating the efficacy of a sun protection tool because they are mostly triggered by UV exposure, are predictive of SCC incidence, and are 10-20x more prevalent than NMSC. ${ }^{14}$

We did not observe any significant difference between the two groups in the incidence rate ratio of AKs (0.799) over 3 months between disenrollment at 6 months and the intermediary visit at 3 months $(p$-value $=0.44,95 \% \mathrm{Cl}:[0.450,1.415])$. One possible explanation is that the control group also changed its behavior because of the dermatologist's active counseling. This is suggested by observing a non-significant decrease in the incidence rate over 3 months for the control group $(-0.3$ AKs per 3 months, p-value $=0.7)$ and the device group $(-1.5$ AK per 3 month, p-value $=0.13$ ). Although this decrease can be accounted for by the seasonal changes in UV, we observed a significant increase of 0.176 ( $p$-value $=0.01$ ) in the incidence rate of NMSC over 3 months for the control group. In Thompson's study, ${ }^{15}$ which similarly enrolled participants in Australia with a history of AKs and the same mean age (63 years old), they measured a decrease of the mean by 0.6 AKs in the sunscreen group over 6 months, suggesting that patients in our study's control group applied sunscreen.

We measured an IRR of 0.05 (p-value $=0.024,95 \% \mathrm{Cl}:[0.004,0.67]$ ) of newly-formed NMSC over 3 months between study termination at 6 months and the intermediary visit at 3 months (Table 2). The separate numbers of BCC and SCC were too small to compute an IRR. One surprising result of our study is the rapid impact the device had on NMSC, which might be explained by two molecular mechanisms. The first one is related to the biology of p53 immunopositive epidermal keratinocytes (p53 "patches"). These p53 patches follow UVR exposure ${ }^{16}$ and are associated with carcinoma of the skin, with $50 \%$ of all skin cancers expressing these mutations. ${ }^{17,18}$ The prevalence of p53 patches increases with age until saturation when people reach the age of 60 years old. ${ }^{19}$ Using a murine 
model, Rebel et al. showed that SCC start appearing immediately after p53 patch saturation and their count grows exponentially with time when mice continue to be exposed to daily UVR. ${ }^{20}$ These data suggest that short-term UV exposure may trigger the appearance of NMSC, after the skin is saturated with p53 patches, as observed in this study. The second molecular mechanism is related to UV-induced immunosuppression, known to trigger a rapid development of NMSC in transplant recipients because of their immunosuppressive treatments. ${ }^{21}$ Together, these biological mechanisms provide a rationale for the rapid development of NMSC in a population with a history of AKs.

Looking at device usage, the two NMSCs reported at disenrollment in the device group were diagnosed in participants who stopped using the device after a few days, providing additional evidence of the impact of the UV dosimeter on NMSC prevention. In Figure 3, we show that the device group participants who had more than one AK at disenrollment experienced a daily average of 0.60 SED across August and September whereas participants who had 0 or 1 AK experienced a daily average of 0.33 SED across August and September $(p=0.0354$, Welch's t-test). This suggests that a sub-erythema chronic exposure beyond 0.34 SED may contribute to the appearance of AKs over 3 months. Additional evaluation is needed, as this was not the primary endpoint studied.

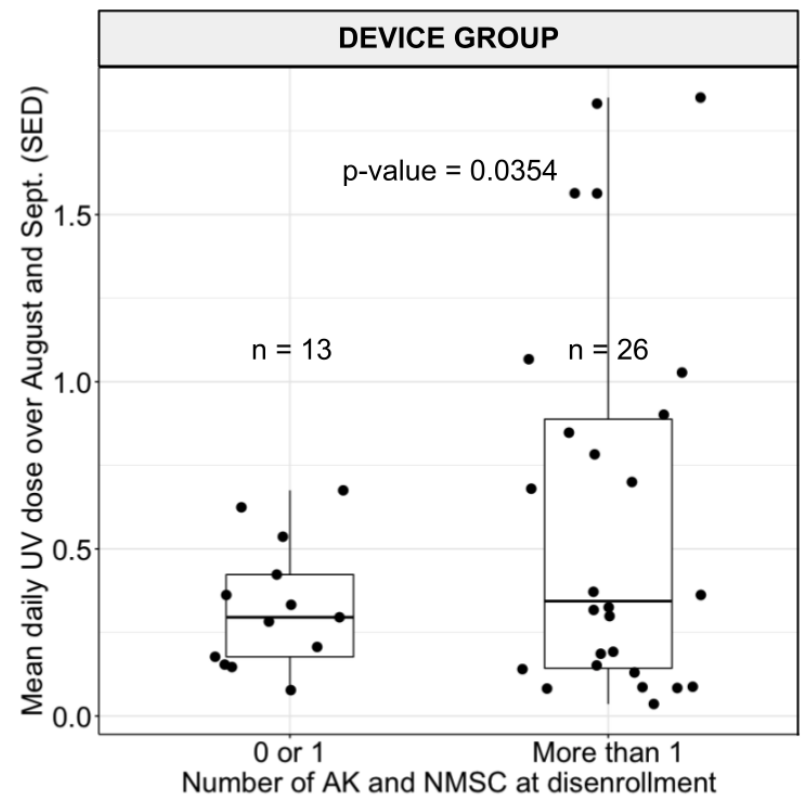

Figure 3. Distribution of the mean daily UV exposure over August and September as a function of the number of AKs measured at disenrollment among patients who wore the UV dosimeter. Using Welch's 2-sample t-test, we found that the group with a low number of AKs had a mean UV exposure of 0.33 SED and the group with a high number of AKs had a mean UV exposure of 0.60 SED ( $p$-value = 0.0354).

We also found that the change in participants' self-perceived ability to participate in social activities, as measured by the PROMIS short-form questionnaire, differed by 2.1 points ( $p$-value $=0.01,95 \% \mathrm{Cl}$ : $[3.67,0.48])$ between disenrollment and enrollment between groups. However, in the control group, the score significantly increased by $1.2(p$-value $=0.04)$ while in the device group, the score non-significantly decreased by 0.9 ( $p$-value $=0.1$ ). This is consistent with a possible growing awareness of the need to moderate UV exposure in the device group and a growing overconfidence 
after repeat UV counseling in the absence of data in the control group. In addition, we did not observe significant changes, or between group differences, in self-reported anxiety and depression levels.

There are several limitations to this study. First, the number of NMSCs is low, which is why we did not present the analysis of SCC and BCC separately. However, their IRRs were also significant ( $p$-value < $1 \mathrm{e}-5$ for SCC and p-value < 1.e-5 for BCC) between disenrollment and the intermediary visit. Second, the study population came from a single recruiting site in New York where UVR is lower than Australia, where similar studies were conducted. We would therefore expect to see a larger impact of the device in higher UVR regions. Third, over $85 \%$ of our participants completed college, twice the national average; however, the unadjusted incidence rates of NMSC at disenrollment in the device and control groups stratified by education are similar $(0.05$ for the device group and 0.24 for the control group for participants who completed college; and 0 for the device group and 0.2 for the control group for participants who did not complete college). Fourth, the population was followed for one summer only, leaving the possibility that the device's impact is short-lived. However, UV dosimeter use is seasonal, just like sunscreen, and we would expect to see an uptake in usage in the Spring. Fifth, we do not know the UV exposure behavior in the control group. We could have given UV dosimeters without access to real-time data to the control group but we chose not to influence control patients' behavior.

Over the past few years, consumers have been able to learn more about their health through the use of sensors in wearable devices (e.g., smartwatches now measure vital signs). This clinical trial is the first to provide evidence of the clinical efficacy of an accurate wearable UV sensor in skin cancer prevention. 


\section{REFERENCES}

1. Garbe C, Keim U, Gandini S, et al. Epidemiology of cutaneous melanoma and keratinocyte cancer in white populations 1943-2036. Eur J Cancer 2021;152:18-25.

2. Samarasinghe V, Madan V. Nonmelanoma skin cancer. J Cutan Aesthet Surg 2012;5(1):3.

3. Robinson JK, Rademaker AW, Sylvester JA, Cook B. Summer sun exposure: knowledge, attitudes, and behaviors of Midwest adolescents. Prev Med 1997;26(3):364-72.

4. Waldman RA, Grant-Kels JM. The role of sunscreen in the prevention of cutaneous melanoma and nonmelanoma skin cancer. J Am Acad Dermatol 2019;80(2):574-6.e1.

5. Linos E, Keiser E, Fu T, Colditz G, Chen S, Tang JY. Hat, shade, long sleeves, or sunscreen? Rethinking US sun protection messages based on their relative effectiveness. Cancer Causes Control 2011;22(7):1067-71.

6. Chen AC, Martin AJ, Choy B, et al. A Phase 3 Randomized Trial of Nicotinamide for Skin-Cancer Chemoprevention. N Engl J Med 2015;373(17):1618-26.

7. Merhi S, Salameh P, Kaplan P, et al. An Ecological Study Indicates the Importance of Ultraviolet A Protection in Sunscreens. Acta Derm Venereol 2021;101(6):adv00480.

8. Matta MK, Zusterzeel R, Pilli NR, et al. Effect of Sunscreen Application Under Maximal Use Conditions on Plasma Concentration of Sunscreen Active Ingredients: A Randomized Clinical Trial. JAMA 2019;321(21):2082-91.

9. Corrêa MDP, Godin-Beekmann S, Haeffelin M, et al. Comparison between UV index measurements performed by research-grade and consumer-products instruments. Photochem Photobiol Sci 2010;9(4):459-63.

10. Banerjee S, Hoch EG, Kaplan PD, Dumont ELP. A comparative study of wearable ultraviolet radiometers. In: 2017 IEEE Life Sciences Conference (LSC). 2017. p. 9-12.

11. Marks R. Nnmelanotic skin cancer and solar keratoses: The quiet 20th century epidemic. Int J Dermatol 1987;26(4):201-5.

12. Marks R, Foley P, Goodman G, Hage BH, Selwood TS. Spontaneous remission of solar keratoses: the case for conservative management. Br J Dermatol 1986;115(6):649-55.

13. Institute NC, National Cancer Institute. Fitzpatrick Classification Scale [Internet]. Definitions. 2020;Available from: http://dx.doi.org/10.32388/3lujmq

14. Marques E, Chen TM. Actinic Keratosis. In: StatPearls. Treasure Island (FL): StatPearls Publishing; 2021.

15. Thompson SC, Jolley D, Marks R. Reduction of solar keratoses by regular sunscreen use. N Engl J Med 1993;329(16):1147-51.

16. Brash DE, Rudolph JA, Simon JA, et al. A role for sunlight in skin cancer: UV-induced p53 mutations in squamous cell carcinoma. Proc Natl Acad Sci U S A 1991;88(22):10124-8.

17. Boukamp P. Non-melanoma skin cancer: what drives tumor development and progression? Carcinogenesis 2005;26(10):1657-67.

18. Giglia-Mari G, Sarasin A. TP53 mutations in human skin cancers. Hum Mutat 2003;21(3):217-28.

19. Grandi V, di Gennaro P, Torrigiani S, Basco L, Lastrucci I, Pimpinelli N. Ingenol mebutate-mediated reduction in p53-positive keratinocytes in skin cancerization field directly correlates with clinical response in patients with multiple actinic keratoses. J Eur Acad Dermatol Venereol 2019;33(7):1297-303.

20. Rebel H, Kram N, Westerman A, Banus S, van Kranen HJ, de Gruijl FR. Relationship between UV-induced mutant p53 patches and skin tumours, analysed by mutation spectra and by induction kinetics in various DNA-repair-deficient mice. Carcinogenesis 2005;26(12):2123-30.

21. Rangwala S, Tsai KY. Roles of the immune system in skin cancer. Br J Dermatol 2011;165(5):953-65. 